American Journal of Immunology 3 (1) : 4-9, 2007

ISSN 1553-619X

(C) 2007 Science Publications

\title{
Protection against Lipopolysaccharide-induced Death by An Anti-Interleukin-6 Monoclonal Antibody
}

\author{
${ }^{1}$ Bailin Liang, ${ }^{1}$ Debra Gardner, ${ }^{1}$ Don Griswold and ${ }^{2}$ Xiao-yu R. Song \\ ${ }^{1}$ Immunobiology, Centocor, 145 King of Prussia Road, Radnor, PA 19087 \\ ${ }^{2}$ Ethicon, Research \& Development, Route 22 West, P.O. Box 151, Somerville, NJ 08876-0151
}

\begin{abstract}
Septicemia is frequently associated with serious complications and high mortality despite recent advances in treatment with antibacterial agents. Infectious organisms or their soluble products initiate septic shock through a complex cytokine cascade. Lipopolysaccharide (LPS) induced excessive production of inflammatory cytokines is regarded as a model of septic shock. Experiments of LPSinduced excessive cytokine production were conducted in Balb/c mice for the investigation of the therapeutic potential of anti-TNF- $\alpha$ and anti-IL-6 monoclonal antibody treatments. LPS injection resulted in mortality with significant serum levels of TNF- $\alpha$, IL-6, and IL-1 $\beta$. Anti-IL-6 treatment significantly reduced animal mortality and inhibited serum levels of IL- 6 . Anti-TNF- $\alpha$ treatment did not affect serum IL-6 levels even though it significantly enhanced animal survival and inhibited TNF- $\alpha$ production. Moreover, anti-IL-6 treatment modestly reduced serum TNF- $\alpha$ level in comparison with control antibody treated group. These data suggest that TNF- $\alpha$ and IL-6 may play distinct protective roles in septic shock.
\end{abstract}

Key words: Septic shock, cytokine, mortality, anti-IL-6 antibody, IL-6, TNF- $\alpha$

\section{INTRODUCTION}

Endotoxin-induced septic shock is a leading cause of morbidity and mortality among hospitalized patients, and there is limited treatment currently available despite numerous mechanistic studies of the disease in the last two decades ${ }^{[1]}$. Gram-negative bacteria have been suggested to be a common cause of septic shock, and lipopolysaccharide (LPS), a major constituent of Gram-negative bacterial outer membrane, is believed to be one of the primary mediators of septic shock. LPS and other bacterial constituents interact with proteins such as plasma lipoproteins and LPS binding protein in the circulation resulting in complexes that have high affinity for the opsonic receptor CD14, which then signals through the toll-like receptor 4 (TLR-4)-MD2 complex on monocyte/macrophages ${ }^{[2]}$. This interaction occurs on the surface of mononuclear cells and triggers the activation of monocyte/macrophages and a wide range of inflammatory responses including over production of proinflammatory cytokines such as tumor necrosis factor (TNF)- $\alpha$, interleukin (IL)-6, and interleukin (IL)-1 ${ }^{[1,2]}$; activation of the L-argininenitric oxide (NO) pathway and formation of oxidative free radicals $^{[3]}$; activation of the coagulation cascade, fibrinolysis, production of lipid mediators, and complement pathway ${ }^{[4]}$. All these complications lead to the multiple organ injury that occurs during endotoxic shock.

IL-6 is a pleiotropic cytokine with a variety of biological effects acting through specific gp80 and gp130 membrane receptors that mediate intracellular signal transduction ${ }^{[5]}$. TNF- $\alpha$, interleukin (IL)- 8 , and neutrophil degranulation were not affected by elimination of IL-6 in endotoxemia model of chimpanzees ${ }^{[6]}$. On the contrary, serum IL-6 levels were influenced by TNF- $\alpha$ infusion in humans ${ }^{[7]}$. It has been well established that serum IL-6 levels are greatly increased in sepsis patients ${ }^{[8]}$. Anti-TNF $\alpha$ treatment affected the cytokine expression in the plasma and myocardium in mice exposed to LPS. However, if the elevated myocardial expression of IL- 6 and JE was not successfully controlled, anti-TNF $\alpha$ therapy many not be effective in the treatment of endotoxin shock ${ }^{[2]}$. Overall, the outcomes from various experimental septic shock models are controversial, and the underlying mechanism remains unclear. Recent studies indicated that IL-6 induced high C5a receptor levels in solid organs of murine sepsis and anti-IL-6 treatment significantly reduced mortality ${ }^{[4,9]}$.

The current studies were designed to investigate the therapeutic potential of anti-IL-6 monoclonal

Corresponding Author: $\quad$ Bailin Liang, Ph.D. Department of Immunobiology, Centocor, 145 King of Prussia Road, Radnor, PA. 19087, USA 
antibody treatments for LPS-induced excessive cytokine production in Balb/c mice. Our studies confirmed that anti-TNF- $\alpha$ treatment significantly enhanced animal survival and inhibited TNF- $\alpha$ production, but it did not significantly affect serum IL-6 level. Our studies demonstrated that anti-IL-6 treatment significantly inhibited serum levels of IL-6 and modestly reduced TNF- $\alpha$ level and subsequent animal mortality. Our data suggest that anti-TNF- $\alpha$ and antiIL-6 may have therapeutic utility and suggest distinct roles for both cytokines in septic shock.

\section{MATERIALS AND METHODS}

Antibodies and reagents: $\mathrm{Rat} \mathrm{IgG}$ control $\mathrm{mAb}$ was purchased from Jackson Immunoresearch (West Grove, PA). Rat anti-mouse TNF- $\alpha$ Ab (Lot\# 4922) and Rat anti-mouse IL-6 Ab (Lot\# 5282305) were made by Centocor Cell Biology Lab (Centocor, Radnor, PA).

Animals and experimental protocol: Five to seven weeks old Balb/c mice were obtained from Charles River Laboratories (Wilminton, MA). On day 0, the study animals were randomly assigned to test groups or a control group (15 mice/group). Animals were administered an intraperitoneal injection of control article or different monoclonal antibody (mAb) injections and approximately 1 hour later received an intravenous injection of LPS to induce septic shock. All animals were assessed four times daily for morbidity for 26 hours post-LPS injection. Blood was collected at 4 hours post-LPS injection.

Cytokine analysis by luminex: Analysis of serum cytokine levels was performed by incubation of $25 \mu \mathrm{l}$ of serum with immobilized bead bound antibodies in a 16plex (Linco Research Inc., St. Charles, MO) Luminex assay for quantitative determination of 16 cytokines according to the manufacturer's recommendations. Briefly, samples were harvested 4 hours or 24 hours after LPS injection and incubated with immobilized antibodies overnight at $4^{\circ} \mathrm{C}$, washed and detected by incubation with biotinylated detection antibody and streptavidin-phycoerythrin. Samples were read on a Luminex 100 (Luminex Corporation, Austin, TX). Data were analyzed using nonlinear fit equation.

Serum amyloid A (SAA) analysis by ELISA: Serum SAA levels were determined by ELISA (Biosource, Camarillo, CA) according to the manufacturer's recommendations. Briefly, serum samples were harvested at 4 hours after LPS injection and diluted
1:1000 in assay diluent and incubated with conjugated anti-mouse SAA. Substrate tetramethylbenzidine was added and samples were read at $450 \mathrm{~nm}$ and analyzed using 4 parameter fit to determine sample values.

RNA isolation and detection of C5r mRNA by real time PCR analysis: Lung, heart, liver, and kidney from 5 random animals of each group were harvested 4 hours after LPS injection. Total RNA was isolated from lung, heart, liver, and kidney tissues using the RNeasy Mini Kit (Qiagen Inc., Valencia, CA) according to the manufacturer's recommendations. Quality and quantity of RNA were assessed by Bioanalyzer (Agilent Technologies, Palo Alto, CA). Reverse transcription was performed with $2 \mu \mathrm{g}$ of RNA using Reverse Transription Reagent kit (Applied Biosystems, Foster City, CA) based on manufacturer's recommendations.

Real time PCR analysis was performed with $40 \mathrm{ng}$ cDNA template using C5r primer/probe and Master Mix (Applied Biosystems) in a total of $20 \mu \mathrm{l}$ reaction on the 7900HT (Applied Biosystems) according to the manufacture's recommendations. GAPDH expression was also analyzed as a housekeeping gene using GAPDH primer/probe (Applied Biosystems). Duplicate samples were assayed and C5r levels in all treatment groups were normalized against GAPDH to yield $\Delta \mathrm{Ct}$, and then further normalized against a normal tissue sample to yield $\Delta \Delta \mathrm{Ct}$. Fold change of gene expression was calculated with the formula: $2^{-\Delta \Delta \mathrm{Ct}}$.

Statistical analysis: Animal survival was expressed in groups, various serum cytokines and fold changes of C5r gene expression among various groups were expressed as individual plot for each animal with relevant mean. Statistical significance was determined by two-tailed analysis of variance by standard t-test.

\section{RESULTS}

Animal survival: To determine the effect of anti-IL-6 $\mathrm{mAb}$ treatment in septic shock, survival rates of animals that received anti-IL-6 mAb treatment was compared to animals that received normal rat $\mathrm{IgG}$ control $\mathrm{mAb}$ after LPS induction of septic shock. Figure 1 shows the percentage of animals that survived for at least 26 hours post-LPS injection in each group. There were no survivors in the group of animals, which received normal rat IgG, 24 hours after LPS injection (Fig. 1). There was no additional mortality among the animals in the anti-IL-6 and anti-TNF $\alpha$ treatment 


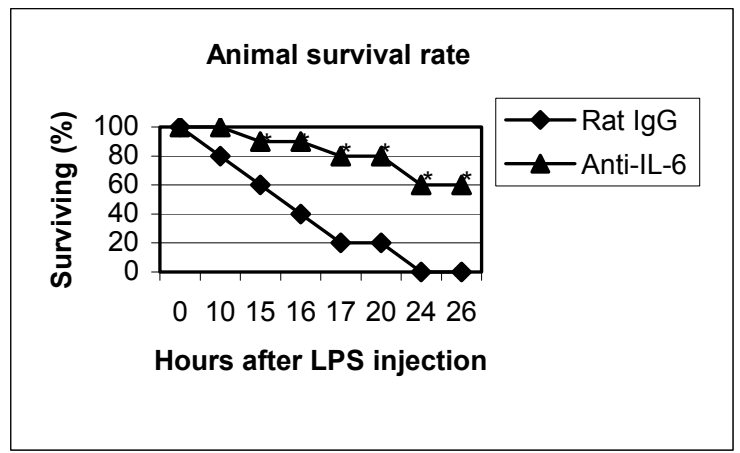

Fig. 1: Anti-IL-6 significantly reduced LPS-induced mortality. Number of survived animals after LPS injection was recorded at various time points and converted to percentage using total number of animals (15 mice) for each group. * indicates $p<0.05$ as compared to the rat IgG-treated group.

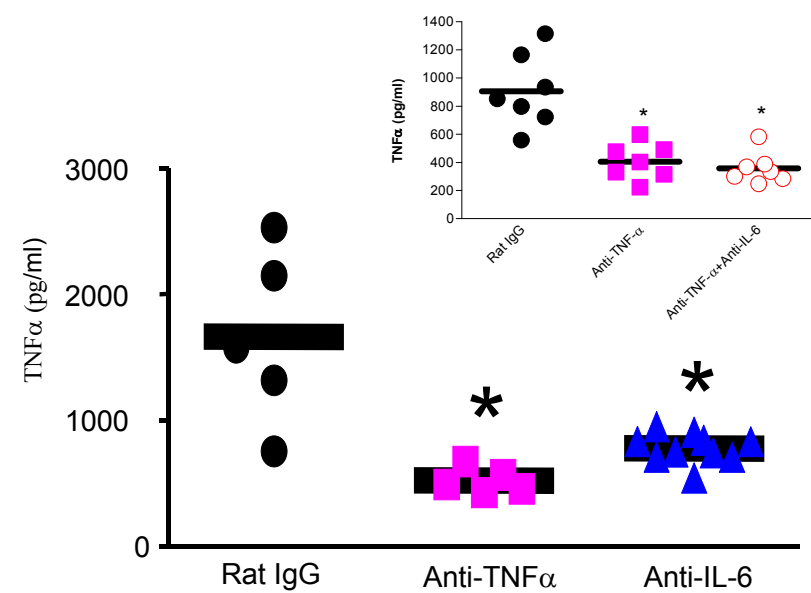

Fig. 2a: Anti-TNF- $\alpha$ and anti-IL-6 inhibited TNF- $\alpha$ production. Serum samples were collected from different groups of animals as indicated 4 hours after LPS injection. Levels of TNF- $\alpha$ were determined by Luminex and expressed as means. The insert represents data from a separate study in which combination of anti-TNF- $\alpha$ and anti-IL-6 mAbs was compared with single mAb treatments. Samples were collected and assayed as in Fig. 2a. * indicates $p<0.05$ as compared to the rat IgG-treated group.

groups between 26-72 hours post-LPS injection (data not shown). Anti-IL-6 treatment significantly improved animal survival (Fig 1), and anti-TNF- $\alpha$ treatment also significantly reduced animal mortality as reported previously (data not shown). These results suggest that IL-6 and TNF- $\alpha$ play important roles in LPS-induced septic shock.

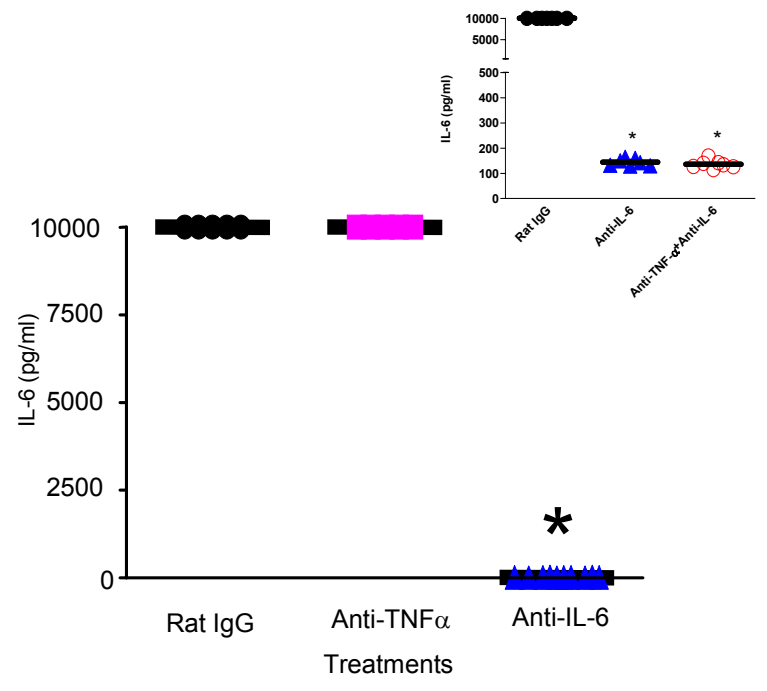

Fig. 2b: Anti-TNF- $\alpha$ and anti-IL-6 inhibited IL-6 production. Serum samples were collected from different groups of animals as indicated 4 hours after LPS injection. Levels of IL-6 were determined by Luminex and expressed as means. The insert represents data from a separate study in which combination of anti-TNF- $\alpha$ and anti-IL- 6 mAbs was compared with single mAb treatments. Samples were collected and assayed as in Fig. 2b. * indicates $p<0.05$ as compared to the rat IgG-treated group.

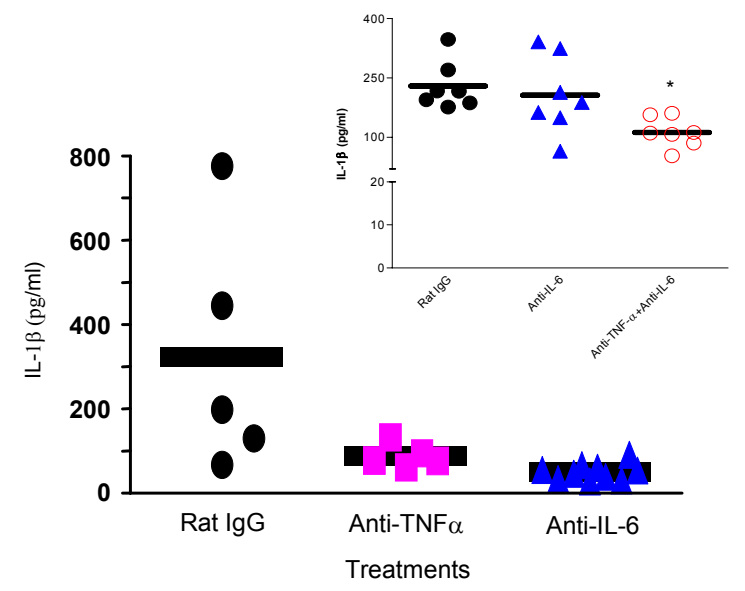

Fig. 2c: Anti-TNF- $\alpha$ and anti-IL-6 inhibited IL$1 \beta$ production. Serum samples were collected from different treatment groups of animals as indicated 4 hours after LPS injection. Levels of IL-1 $\beta$ were determined by Luminex and expressed as means. The insert represents data from a separate study in which combination of anti-TNF- $\alpha$ and anti-IL-6 $\mathrm{mAbs}$ was compared with single $\mathrm{mAb}$ treatments. Samples were collected and assayed as in Fig. 2c. * indicates $p<0.05$ as compared to the rat $\operatorname{IgG}$ and anti-IL-6 treated groups. 


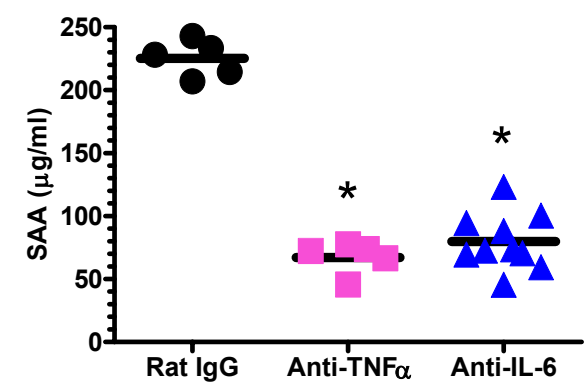

Fig. 3: Anti-TNF- $\alpha$ and anti-IL-6 treatments reduced SAA production. Serum samples were collected from different groups of animals 4 hours after LPS injection. Levels of SAA were determined by ELISA and expressed as means. * indicates $p<0.05$ as compared to the rat IgG treated group.

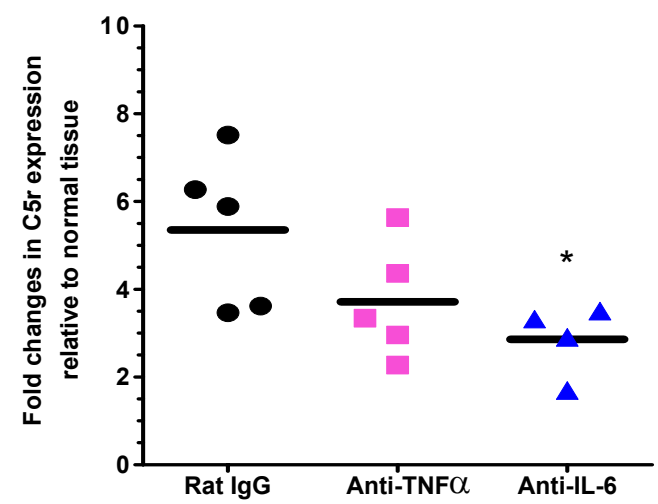

Fig. 4a: Anti-TNF- $\alpha$ and anti-IL-6 reduced C5r expression in the lung. Lung tissues were harvested from 5 random animals of different groups 4 hours after LPS injection. Levels of C5r expression in diseased tissues were determined by real time RT-PCR and compared to that of the normal tissues. * indicates $\mathrm{p}<0.05$ as compared to the rat IgG and anti-TNF $\alpha$ treatment groups.

Serum levels of TNF- $\alpha$, IL-6, and IL-1 $\beta$ : Serum levels of TNF- $\alpha$ and IL- 6 were then measured to investigate the effectiveness of anti-TNF- $\alpha$ and anti-IL$6 \mathrm{mAbs}$ in vivo. Not surprisingly, LPS injection significantly increased serum TNF- $\alpha$, IL- 6 , and IL-1 $\beta$ levels (Fig2a-c). Anti-TNF- $\alpha$ and anti-IL-6 treatments significantly inhibited serum TNF- $\alpha$ and IL- 6 levels, respectively, at $4 \mathrm{hrs}$ after LPS injection as compared to control $\mathrm{Ab}$ treatment group (Fig. 2a, 2b). In addition, anti-IL-6 treatment significantly inhibited serum and TNF- $\alpha$ level. In contrast, anti-TNF- $\alpha$ treatment did not affect serum IL-6 level. In a separate study where a sub-lethal dose of LPS was used, the combination of

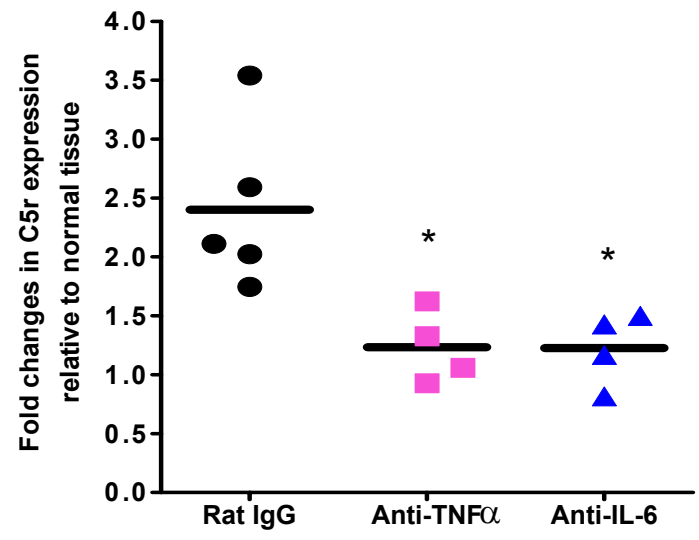

Fig. 4b: Anti-TNF- $\alpha$ and anti-IL-6 reduced C5r expression in the heart. Heart samples were harvested from 4-5 random animals of different groups 4 hours after LPS injection. Levels of C5r expression in diseased tissues were determined by real time RT-PCR and compared to that of the normal tissues. ${ }^{*}$ indicates $\mathrm{p}<0.05$ as compared to the rat IgG treatment group.

anti-TNF- $\alpha$ and anti-IL-6 treatment significantly inhibited serum TNF- $\alpha$ level that was comparable to that of anti-TNF- $\alpha$ treatment alone (Fig. 2a insert). In addition, the combination treatment showed similar inhibitory effects on serum IL-6, level as anti-IL-6 treatment alone (Fig. 2b insert). The combination treatment significantly inhibited serum IL-1ß level as compared to rat IgG and anti-IL-6 treatment alone (Fig. $2 c)$.

\section{Serum amyloid A Levels.}

The major acute-phase protein, serum amyloid A (SAA), is upregulated by a variety of inflammatory stimuli, including cytokines and glucocorticoids. Elevated systemic concentrations of SAA, IL-6, and TNF- $\alpha$ are features of an ongoing inflammatory response. Treatment with anti-TNF- $\alpha$ and anti-IL-6 mAbs resulted in a decreased level of serum SAA (Fig. 3 ) indicating the treatments inhibited the elevated inflammation induced by LPS.

C5r expression in tissues: $\mathrm{C} 5 \mathrm{r}$ expression has been shown to be associated with IL-6 level in various inflammatory tissues. $\mathrm{C} 5 \mathrm{r}$ expression was examined in lung, heart, liver, and kidney 4 hours post-LPS administration. Anti-IL-6 treatment significantly inhibited $\mathrm{C} 5 \mathrm{r}$ expression in the lung and heart (Fig. 4a and $4 \mathrm{~b}$ ), while neither anti-IL-6 nor anti-TNF $\alpha$ significantly affected $\mathrm{C} 5 \mathrm{r}$ expression in the liver and kidney (data not shown). 


\section{DISCUSSION}

Leukocyte recruitment in various tissues and the subsequent dysregulation of proinflammatory responses contribute to the elevated levels of TNF- $\alpha$, IL-6, and IL-1ß as well as mortality in septic shock. The suppression of these proflammatory cytokines with pharmacological strategies or genetic manipulation significantly dampens the hyperpathophysiological responses and protects animals from septic death ${ }^{[10]}$. In our present study, LPS injection induced significant production of TNF- $\alpha$, IL-6, and IL-1 $1 \beta$, which was associated with high mortality in Balb/c mice. AntiTNF- $\alpha$ and anti-IL- 6 mAb treatments significantly reduced LPS-induced mortality in these mice. Anti-IL-6 treatment not only significantly reduced serum IL-6 level, but also reduced serum TNF- $\alpha$ level in mice injected with LPS. Anti-TNF- $\alpha$ and anti-IL-6 may impact independent pathways in LPS-induced septic shock since neither synergistic nor additive effects were observed with combination treatment.

In addition to proinflammatory cytokines, recent studies suggested that complement $\mathrm{C} 5$ and its receptor C5r appeared to be associated with IL-6 in the inflammatory responses occurred in a mouse sepsis model ${ }^{[4,9]}$. C5r was shown to be upregulated in various organs, such as heart, kidney, and liver, by the elevated level of IL-6. Anti-IL-6 mAb treatment significantly suppressed $\mathrm{C} 5 \mathrm{r}$ expression and improved animal survival. In our study, anti-IL-6 mAb treatment significantly reduced $\mathrm{C} 5 \mathrm{r}$ expression in the lung and heart tissues. Furthermore, a series of in vitro studies with endothelial cells demonstrated that C5 could induce significant activation of genes associated with inflammation, i.e. adhesion molecules, proinflammatory cytokines as well as chemokines ${ }^{[11]}$. Conversely, proinflammatory molecules, such as IL-6, could induce C5r expression in endothelial cells ${ }^{[12]}$. This elevated IL-6 level can subsequently sets off a vicious cycle since in vitro stimulation of human umbilical vein endothelial cells with human C5 resulted in progressive increases in gene expression of adhesion molecules and cytokines ${ }^{[11]}$. Mouse dermal microvascular endothelial cells cultured with IL-6 had increased C5 mRNA expression as well as protein expression on the cell surface ${ }^{[12]}$. In our study, anti-IL-6 mAb treatment significantly reduced serum TNF- $\alpha$ level while antiTNF- $\alpha$ mAb treatment did not affect serum IL-6 level in our study.
Combination treatment of anti-TNF- $\alpha$ and anti-IL6 did not further inhibit serum TNF- $\alpha$ and IL-6 levels as compared to anti-TNF- $\alpha$ treatment and anti-IL- 6 treatment alone, respectively. Only the combination treatment significantly inhibited serum IL- $1 \beta$ level as compared to rat IgG and anti-IL-6 treatment alone. These results suggest that TNF- $\alpha$ and IL- 6 play distinct protective roles in septic shock. Not only did anti-IL-6 $\mathrm{mAb}$ treatment directly suppress the elevated IL-6 level in septic shock, but the treatment also indirectly inhibited C5 and C5r levels, which resulted in lower overall inflammatory responses including TNF- $\alpha$ production. On the other hand, anti-TNF- $\alpha$ treatment only inhibited the elevated TNF- $\alpha$ level; it did not suppress the elevated $\mathrm{C} 5 \mathrm{r}$ level in the lung. In conclusion, conventional approach using antiproinflammatory cytokine antibody may not be the best strategy for septic shock treatment. Our study along with other recent reports suggest that antiproinflammatory cytokine treatment that also inhibit complement activation results in higher animal survival and lower inflammation in mouse septic shock models. More studies are needed to fully clarify the mechanisms.

\section{REFERENCES}

1. Ikezoe, T., Y. Yang, D. Heber, H. Taguchi and H.P. Koeffler, 2003. PC-SPES: A potent inhibitor of nuclear factor- $\mathrm{kB}$ rescues mice from lipopolysaccharide-induced septic shock. Mol. Pharmacol., 64: 1521-1529.

2. Kadokami, T., C.F. McTiernan, T. Kubota, C. Frye, G.S. Bounoutas, P.D. Robbins, S.C. Watkins and A.M. Feldman, 2001. Effects of soluble TNF receptor treatment on lipopolysaccharide-induced myocardial cytokine expression. Am. J. of Physiol - Heart and Circulatory Physiology, 280: H22812291.

3. Boveris, A., S. Alvarez and A. Navarro, 2002. The role of mitochondrial nitric oxide synthase in inflammation and septic shock. Free Radical Bio. Med., 33: 1186-1193.

4. Riedemann, N.C., T.A. Neff, R-F Guo, K.D. Bernacki, I.J. Laudes, J.V. Sarma, J.D. Lambris and P.A. Ward, 2003. Protective effects of IL-6 blockade in sepsis are linked to reduced C5a receptor expression. J. Immunol., 170: 503-507. 
5. Boulanger, M.J., D.D. Chow, E.E. Brevnova and K.C. Garcia, 2003. Hexameric structure and assembly of the interleukin-6/IL-6 $\alpha$ receptor/gp130 complex. Science, 300: 2101-2104.

6. van der Poll, T., M. Levi, C.E. Hack, H. ten Cate, S.J.H. van Deventer, A.J.M. Eerenberg, E.R. de Groot, J. Jansen, H. Gallati, H.R. Buller, J.W. ten Cate and L.A. Aarden, 1994. Elimination of IL-6 attenuates coagulation activation in experimental endotoxemia in chimpanzees. J. Exp. Med., 179: 1253-1259.

7. Sheron, N., J.N. Lau, J. Hofmann, R. Williams and G.J. Alexander, 1990. Dose-dependent increase in plasma interleukin-6 after recombinant tumour necrosis factor infusion in humans. Clin. and Exp. Immunol., 82: 427-428.

8. Hack, C.E., E.R. de Groot, R.J. Felt-Bersma, J.H. Nuijens, R.J. Strack Van Schijndel, A.J. Eerenberg-Belmer, L.G. Thijs and L.A. Aarden, 1989. Increased plasma levels of interleukin-6 in sepsis. Blood, 74: 1704-1710.
9. Riedemann, N.C., R.F. Guo, T.A. Neff, I.J. Lauses, K.A. Keller, J.V. Sarma, M.M. Markiewski, D. Mastellos, C.W. Strey, C.L. Pierson, J.D. Lambris, F.S. Zetoune and P.A. Ward, 2002. Increased C5a receptor expression in sepsis. J. Clin. Invest., 110: 101-108.

10. Joshi, V.D., D.V. Kalvakolanu and A.S. Cross, 2003. Simultaneous activation of apoptosis and inflammation in pathogenesis of septic shock: a hypothesis. FEBS Letters, 555: 180-184.

11. Albrecht, E.A., A.M. Chinnaiyan, S. Varambally, C. Kumar-Sinha, T.R. Barrette, J.V. Sarma and P.A. Ward, 2004. C5a-induced gene expression in human umbilical vein endothelial cells. Am. J. Pathol., 164: 849-859.

12. Lauses, I.J., J.C. Chu, M. Huber-Lang, R.F. Guo, N.C. Riedemann, J.V. Sarma, F. Mahdi, H.S. Murphy, C. Speyer, K.T. Lu, J.D. Lambris, F.S. Zetoune and P.A. Ward, 2002. Expression and function of $\mathrm{C} 5 \mathrm{a}$ receptor in mouse microvascular endothelial cells. J. Immunol., 169: 5962-5970. 